\title{
An improved formal total synthesis of (-)-anisomycin
}

\author{
Pei -Qiang Huang*, Xiao Zheng \\ Department of Chemistry, Xiamen University, Xiamen, Fujian 361005, CHINA \\ E-mail:pqhuang@xmu.edu.cn
}

\section{Dedicate with best wishes to Professor Zhi-Tang Huang on the occasion of his 75th birthday}

(received 5 Jul 02; accepted 24 Sep 02; published on the web 26 Sep 02)

\begin{abstract}
(R)-1-Benzyloxycarbonyl-2-(4-methoxyphenyl)methyl-3-pyrroline 14 was synthesized from (S)- $N$, O-dibenzyl malimide 5 via a highly regio and trans stereoselective reductive alkylation of 5. This constitutes an improved formal asymmetric synthesis of natural (-)-anisomycin.
\end{abstract}

Keywords: Anisomycin, malic acid, reductive alkylation, asymmetric synthesis, malimide

\section{Introduction}

Polysubstituted pyrrolidines represent a class of bioactive natural products as exemplified by the antifungal antibiotics (-)-anisomycin 1, (+)-preussin 2 and (-)-codonopsinine 3.

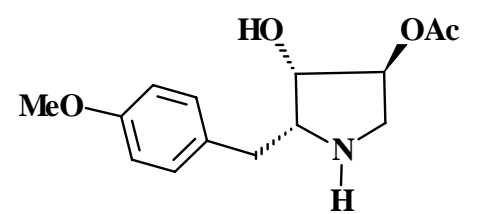

(-)-Anisomycin 1

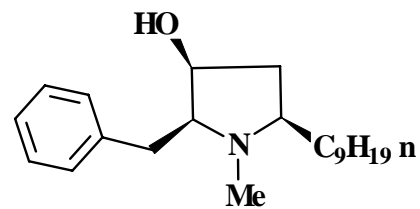

$(+)$-Preussin 2<smiles>COc1ccc(C2N[C@H](C)C(O)C2O)cc1</smiles>

(-)-Codonopsinine 3

Since its first isolation from Streptomyces griseolus and S. roseochromogenes ${ }^{1}$ in the early fifties of last century, anisomycin has become an important tool in molecular biology. ${ }^{2}$ It also has been used for the treatment of trichomonas vaginitis ${ }^{3}$ and amebic dysentery, ${ }^{4}$ and as an agricultural fungicide. ${ }^{5}$ More recently, it was reported that anisomycin showed high in vitro antitumor activity, ${ }^{6}$ and could be used in a synergistic fashion with a cyclin-dependent protein kinase inhibitor to kill carcinoma cells. ${ }^{7}$ Consequently, many approaches have been developed 
for the asymmetric synthesis of anisomycin. ${ }^{8}$ In continuation of our efforts on the development of amalic acid-based methodology to bioactive $\mathrm{N}$-containing compounds, ${ }^{9}$ we have communicated an asymmetric approach to (-)-anisomycin. ${ }^{10}$ In that approach, the key reductive alkylation of $\mathbf{4}$ led to $\mathbf{9}$ in modest regioselectivity. We would like to report herein an improved formal asymmetric synthesis to (-)-anisomycin, which featured the reductive alkylation of (S)-N,O-dibenzyl malimide 5 in high regio- and stereo-selectivity.

\section{Results and Discussion}

The synthesis began with the reductive alkylation of (S)- $N, O$-dibenzyl malimide 5 (Scheme 1), which was prepared starting from $(S)$-malic acid as described previously. Thus, the addition of p-methoxybenzylmagnesium chloride to $\mathbf{5}$ led smoothly to the desired $\alpha$-hydroxylactam $\mathbf{6}$ as a diastereomeric mixture and in high regioselectivity, which was deduced in the followed step. The stereochemistry of the isomeric $\mathbf{6}$ was not assigned. This diastereomeric mixture, although separable by flash chromatography, was used in the next step as it was, since the followed Lewis acid mediated ionic hydrogenation ${ }^{11}$ was considered to proceed by the intermediacy of the $\mathrm{N}$-acyliminium 7.9,12 Indeed, in the presence of 3.0 equivalents of boron trifluoride etherate, hydroxylactams 6 were reduced with excess of triethylsilane $\left(\mathrm{CH}_{2} \mathrm{Cl}_{2},-78^{\circ} \mathrm{C} \sim\right.$ r.t. $)$ to yield predominantly trans-8 in $94.8 \%$ yield. The coupling constant of $\sim 0 \mathrm{~Hz}$ for $\mathrm{H}-4 / \mathrm{H}-5$ in 8 indicates a trans orientation ${ }^{9}$ of these two protons, was later confirmed by converting 8 to known $(R)-(-)-14^{8 e, f, i}$.

Catalytic hydrogenation $\left(10 \% \mathrm{Pd} / \mathrm{C}, \mathrm{H}_{2}, 1 \mathrm{~atm}\right)$ of $\mathbf{8}$ afforded $\mathbf{9}$ in quantitative yield. 4-Hydroxypyrrolidinone 9 was reduced to pyrrolidine $10\left(\mathrm{LiAlH}_{4}, \mathrm{THF}\right.$, reflux) in 90\% yield. $N$-Debenzylation in the presence of Pearman's catalyst $\left(\mathrm{H}_{2}, \mathrm{Pd}(\mathrm{OH})_{2} / \mathrm{C}, \mathrm{EtOH}\right.$, r.t.) followed by selective $N$-benzyloxycarbonylation ( $\mathrm{CbzCl}, \mathrm{NEt}_{3}, \mathrm{CH}_{2} \mathrm{Cl}_{2}, 0{ }^{\circ} \mathrm{C} \rightarrow$ room temperature) gave $\mathrm{N}$-protected pyrrolidine 12 in an overall yield of $77 \%$. Xanthation of alcohol 12 then provided 13 in $72 \%$ yield. Upon thermolysis of 13 at $190{ }^{\circ} \mathrm{C}$ under reduced pressure, the desired (R)-3-pyrroline $14\left\{[\alpha]_{\mathrm{D}}{ }^{20}-214\left(c 1.18, \mathrm{CHCl}_{3}\right)\right.$. lit. $\left[[\alpha]_{\mathrm{D}}{ }^{20}+1.4\right.$ ( e.e. $=90 \%$ ) for $(S)-14 ;^{8 \mathrm{~h}}[\alpha]_{\mathrm{D}}$ $-190.4\left(c\right.$ 1.0, $\left.\mathrm{CHCl}_{3}\right) ;{ }^{81}[\alpha]_{\mathrm{D}}{ }^{25}-199\left(\right.$ c 1.17, $\left.\mathrm{CHCl}_{3}\right) ;{ }^{8 \mathrm{~m}}[\alpha]_{\mathrm{D}}{ }^{20}-135.7$ (c 0.76, $\left.\mathrm{CHCl}_{3}\right),{ }^{10}$ for $(R)-14]$ \} was obtained in $87.3 \%$ yield, of which the spectroscopic data fully matched those reported in the literature ${ }^{6 m}$.

Since $(+)-\mathbf{1 4}$ has been converted into unnatural enantiomer $(+)$-anisomycin $\mathbf{1}^{8 \mathrm{~b}, \mathrm{l}, \mathrm{m}}$, the present work constitutes a new formal asymmetric synthesis of the natural enantiomer of this antibiotic. 


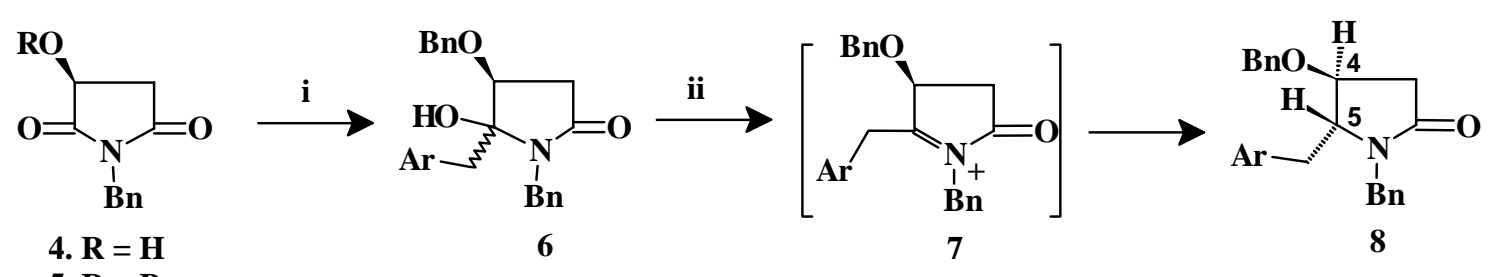

5. $\mathbf{R}=\mathbf{B n}$

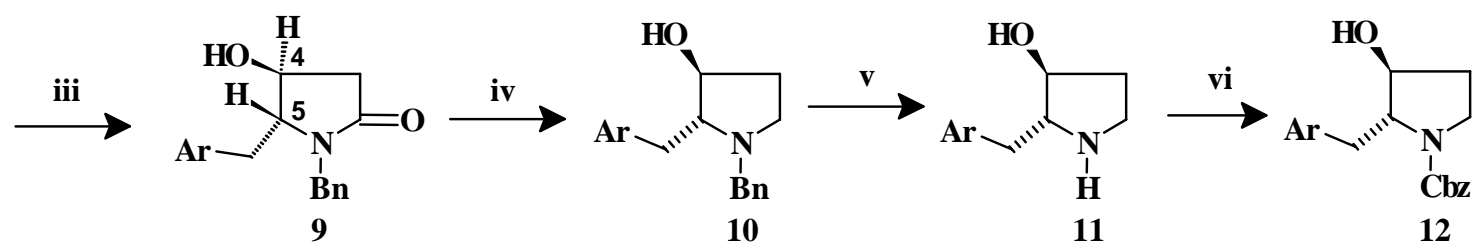

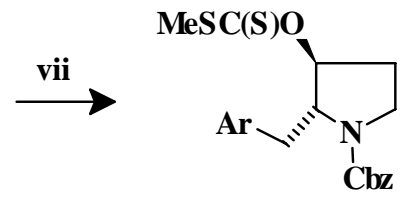

13

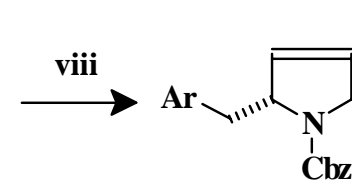

(R)-(-)-14

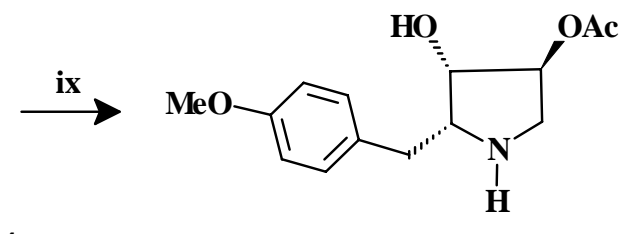

$(-)-1$

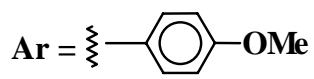

Scheme 1. Reagents and conditions: i) p-MeOC6H4CH2MgCl, THF, $-15{ }^{\circ} \mathrm{C} \sim-10{ }^{\circ} \mathrm{C}, 94.5 \%$; ii) BF3 •OEt2, Et3SiH, -78 ${ }^{\circ} \mathrm{C}, 94.8$ \%; iii) H2, 1 atm, 10 \% Pd/C, EtOH, 100 \%; iv) LiAlH4, THF, reflux, 90 \%; v) H2, 1atm, 20 \% $\mathrm{Pd}(\mathrm{OH}) 2 / \mathrm{C}$, EtOH, r.t.; vi) $\mathrm{CbzCl}$, Et3N, CH2Cl2, r.t., 77 \% over two steps; vii) $\mathrm{NaH}$, THF, imidazole, $65{ }^{\circ} \mathrm{C}$; CS2, $60{ }^{\circ} \mathrm{C}$; MeI, $60{ }^{\circ} \mathrm{C}$, $72 \%$; viii) neat, 190 ${ }^{\circ} \mathrm{C},<10 \mathrm{mmHg}, 87.3 \%$; ix) ref. 8h.

\section{Experimental Section}

General Procedures. Melting points were determined on a Yanaco MP-500 micro melting point apparatus. Infrared spectra were measured with a Shimadza IR-408 spectrometer or a Nicolet Avatar 360 FT-IR spectrometer using film $\mathrm{KBr}$ pellet techniques. ${ }^{1} \mathrm{H}$-NMR spectra were recorded in $\mathrm{CDCl}_{3}$ on a Varian unity +500 spectrometer with tetramethylsilane as an internal standard. Chemical shifts are expressed in $\delta$ (ppm) units downfield from TMS. Mass spectra were recorded by Finnigan Mat-LCQ (ESI direct injection). Optical rotations were measured with Perkin-Elmer 341 automatic polarimeter. THF and diethyl ether used in the reactions were dried by distillation over metallic sodium and benzophenone; dichloromethane were distilled over $\mathrm{P}_{2} \mathrm{O}_{5}$. Silica gel (Zhifu, 300 400 mesh) was used for column chromatography, eluting (unless otherwise stated) with ethyl acetate/petroleum ether $(\mathrm{PE})\left(60-90^{\circ} \mathrm{C}\right)$ mixtures. 
(S)-1-Benzyl-4-benzyloxy-5-(4-methoxybenzyl)-2-pyrrolidinone (8). To a stirred cold solution of (S)-O-Benzyloxy malimide ${ }^{9 a}(2.5 \mathrm{~g}, 8.47 \mathrm{mmol})$ in $\mathrm{THF}(15 \mathrm{~mL})$ at $-15{ }^{\circ} \mathrm{C}$ was added dropwise a 1.0 M solution of freshly prepared 4-methoxybenzylmagnesium chloride in THF (15 $\mathrm{mL}, 15 \mathrm{mmol}$ ) under an atmosphere of $\mathrm{N}_{2}$. The mixture was stirred at $-10 \sim-15{ }^{\circ} \mathrm{C}$ for 1 hour and then quenched by adding a saturated aqueous solution of $\mathrm{NH}_{4} \mathrm{Cl}(15 \mathrm{~mL})$ and water $(10 \mathrm{~mL})$ at $-10{ }^{\circ} \mathrm{C}$. After diluted by dichloromethane $(100 \mathrm{~mL})$ the mixture was poured into a separatory funnel. The orgnic layer were separated, and the aqueous phase was extracted with $\mathrm{CH}_{2} \mathrm{Cl}_{2}(3 \times 25$ $\mathrm{mL}$ ). The combined organic phase was washed with brine, dried with anhydrous $\mathrm{Na}_{2} \mathrm{SO}_{4}$. After flash chromatographic purification (eluent: ethyl acetate: petroleum ether $=1: 2$ ), a white solid (3.34 g, 94.5\%) were obtained which is a mixture of two diastereisoomers. This diastereomeric mixture (2.92 g, $7.0 \mathrm{mmol})$ was dissolved in dry dichloromethane $(28 \mathrm{~mL})$, and cooled to $-78{ }^{\circ} \mathrm{C}$. $\mathrm{Et}_{3} \mathrm{SiH}(11.2 \mathrm{~mL}, 70 \mathrm{mmol})$ and $\mathrm{BF}_{3} \bullet \mathrm{OEt}_{2}(2.6 \mathrm{~mL}, 21 \mathrm{mmol})$ were successively added. The resulting mixture was stirred at $-78^{\circ} \mathrm{C}$ for 6 hours, and then allowed to rise to room temperature. After stirred at room temperature for 6 hours the mixture was quenched by adding a saturated solution of $\mathrm{NaHCO}_{3}(8 \mathrm{~mL})$ at $0{ }^{\circ} \mathrm{C}$. After diluted by dichloromethane $(50 \mathrm{~mL})$, the mixture was poured into a separatory funnel. The orgnic layer were separeted. The aqueous phase was extracted with $\mathrm{CH}_{2} \mathrm{Cl}_{2}(3 \times 10 \mathrm{~mL})$. The combined organic phase was washed with brine, and dried with anhydrous $\mathrm{Na}_{2} \mathrm{SO}_{4}$. After flash chromatographic purification (eluent: ethyl acetate: petroleum ether $=1: 1.5)$, pyrrolidinone 8 was obtained as a colorless oil $(2.66 \mathrm{~g}, 94.8 \%)$. $[\alpha]_{\mathrm{D}}{ }^{20}$ 25.6 (c 0.6, $\mathrm{CHCl}_{3}$ ). IR (film) $v_{\max }$ : 3050, 2930, 2850, 1687, 1614, 1512, 1444, 1302, 1250, 1175, 1070, 1030, 820, 735, $695 \mathrm{~cm}^{-1} .{ }^{1} \mathrm{H}-\mathrm{NMR}\left(500 \mathrm{Mz}, \mathrm{CDCl}_{3}\right) \delta: 2.45$ (m, 2H, H-3), 2.51 (dd, J= 8.48, 13.94 Hz, 1H, H-6), 2.86 (dd, J= 4.52, 13.94 Hz, 1H, H-6), 3.68 (dd, J= 4.52, 8.48 Hz, 1H, $\mathrm{H}-5$ ), 3.80 (s, 3H, $\mathrm{OCH}_{3}$ ), 3.85 (dd, J= 2.07, $4.83 \mathrm{~Hz}, 1 \mathrm{H}, \mathrm{H}-4$ ), 3.96 (d, J= $15.20 \mathrm{~Hz}, 1 \mathrm{H}$, $\left.\mathrm{NCH}_{2} \mathrm{Ph}\right), 4.11\left(\mathrm{~d}, \mathrm{~J}=11.90 \mathrm{~Hz}, 1 \mathrm{H}, \mathrm{OCH}_{2} \mathrm{Ph}\right), 4.16\left(\mathrm{~d}, \mathrm{~J}=11.90 \mathrm{~Hz}, 1 \mathrm{H}, \mathrm{OCH}_{2} \mathrm{Ph}\right), 5.16(\mathrm{~d}, \mathrm{~J}=$ $\left.15.20 \mathrm{~Hz}, 1 \mathrm{H}, \mathrm{NCH}_{2} \mathrm{Ph}\right), 6.80-7.40(4 \mathrm{~m}, 14 \mathrm{H}, \mathrm{Ph}-\mathrm{H}) \mathrm{ppm} ;{ }^{13} \mathrm{C}-\mathrm{NMR}\left(125 \mathrm{Mz}, \mathrm{CDCl}_{3}\right) \delta: 36.08$ (1C), 37.10 (1C), 44.27 (1C), 55.28 (1C), 64.11 (1C), 70.07 (1C), 74.80 (1C), 114.20 (2×Ar-CH), $127.56(3 \times \mathrm{Ar}-\mathrm{CH}), 127.66(1 \times \mathrm{Ar}-\mathrm{CH}), 127.93(3 \times \mathrm{Ar}-\mathrm{CH}), 128.33(2 \times \mathrm{Ar}-\mathrm{CH}), 128.73$

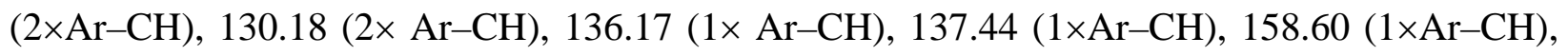
$173.07(\mathrm{C}=\mathrm{O})$ ppm. MS (EI): $401\left(\mathrm{M}^{+}, 2.5\right), 280$ (29), 254 ( 11), 91 (100). HRMS calcd for $\left[\mathrm{C}_{26} \mathrm{H}_{27} \mathrm{O}_{3} \mathrm{~N}\right]^{+}$401.1991, found 401.1983.

(S)-1-Benzyl-4-hydroxy-5-(4-methoxybenzyl)-2-pyrrolidinone (9). To a mixture of 8800 $\mathrm{mg}, 2 \mathrm{mmol})$ and $10 \% \mathrm{Pd}-\mathrm{C}(170 \mathrm{mg})$ was added ethanol $(10 \mathrm{~mL})$. The mixture was stirred at room temperature and under an atmosphere of $\mathrm{H}_{2}$ for 7 days. The mixture was filtered over celite. Flash chromatographic purification on silica gel (eluent: ethyl acetate: petroleum ether $=2: 1$ ) provided pyrrolidinone 9 as a colorless oil $(620 \mathrm{mg}, 100 \%) \cdot[\alpha]_{\mathrm{D}}{ }^{20}+4.80$ (c 1.0, $\left.\mathrm{CHCl}_{3}\right)$. IR (film) $v_{\max }: 3350,2820,1660,1510,1450,1250,1175,1025,690 \mathrm{~cm}^{-1} .{ }^{1} \mathrm{H}-\mathrm{NMR}(500 \mathrm{Mz}$, $\mathrm{CDCl}_{3}$ ) $\delta: 2.27$ (dd, J= 1.07, $17.50 \mathrm{~Hz}, 1 \mathrm{H}, \mathrm{H}-3$ ), 2.45 (dd, J= 6.14, $17.50 \mathrm{~Hz}, 1 \mathrm{H}, \mathrm{H}-3$ ), 2.55 (dd, $\mathrm{J}=$ 7.98, 14.05 Hz, 1H, H-6), 2.86 (dd, J= 4.76, 14.05 Hz, 1H, H-6), 3.50 (dd, J= 4.76, 7.98 Hz, $1 \mathrm{H}, \mathrm{H}-5), 3.78$ (s, 3H, OCH$)_{3}$ ), 3.93 (d, J= $15.20 \mathrm{~Hz}, 1 \mathrm{H}, \mathrm{NCH}_{2} \mathrm{Ph}$ ), 4.16 (s, 1H, H-4), 5.12 (d, J=

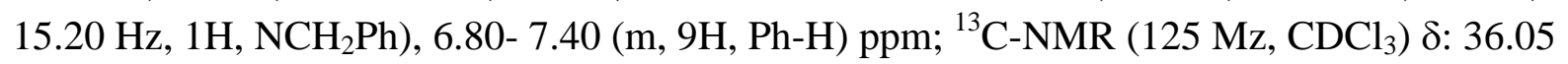


(1C), 39.59 (1C), 44.30 (1C), 55.28 (1C), 67.06 (1C), 68.96 (1C), 114.30 (2×Ar-CH), 127.65

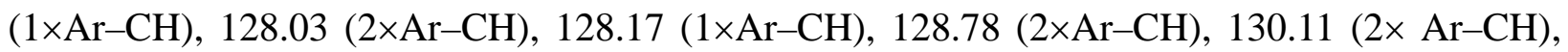
136.16 (1×Ar-CH), 158.65 (1×Ar-CH), $172.88(\mathrm{C}=\mathrm{O})$ ppm. MS (EI): $311\left(\mathrm{M}^{+}, 3.2\right), 293$ (M-18, 43.5), 190 (83.1), 91(100), 57 (99); HRMS calcd for $\left[\mathrm{C}_{19} \mathrm{H}_{19} \mathrm{NO}_{2}\left(\mathrm{M}^{+}-\mathrm{H}_{2} \mathrm{O}\right)\right]$ 293.1416, found 293.1413.

(S)-1-Benzyl-3-hydroxy-2-(4-methoxybenzyl)-pyrrolidine (10). To a ice-cooled suspension of LAH (230 mg, $4.5 \mathrm{mmol}$ ) in anhydrous THF (3.0 mL), was added, under an atmosphere of $\mathrm{N}_{2}$, a solution of 9 (430 mg, $1.38 \mathrm{mmol})$ in THF (4 mL). After stirred at room temperature for 2 hours, then at $50{ }^{\circ} \mathrm{C}$ for 4 hours, the mixture was cooled with a ice-bath, then wet diethyl ether (6 $\mathrm{mL}), 10 \%$ solution of sodium hydroxide $(107 \mathrm{~mL})$ and water $(0.3 \mathrm{~mL})$ were added successively. The mixture was allowed to reach room temperature, stirred for $30 \mathrm{~min}$, and filtered through celite. After concentrated under reduced pressure, the residue was purified by flash chromatography on silica gel ( eluent: ethyl acetate: petroleum ether $: \mathrm{NH}_{3}$ aq. $=2: 1: 0.01$ ). The pyrrolidine 10 (382 mg, yield, 93\%) was abtained as a colorless oil. [ $\alpha]_{\mathrm{D}}{ }^{20}-54.3$ (c 1.0, $\mathrm{CHCl}_{3}$ ). IR (film) $v_{\max }$ : 3450, 2850, 1610, 1510, 1450, 1245, 1175, 1025, $690 \mathrm{~cm}^{-1} .{ }^{1} \mathrm{H}-\mathrm{NMR}$ (500 Mz, $\mathrm{CDCl}_{3}$ ) $\delta: 1.61$ (m, 1H, H-4), 1.99 (m, 1H, H-4), 2.48-2.58 (m, 2H, H-5, H-6), 2.65 (m, 1H, H-5), 2.87 (m, 1H, H-2), 2.95 (dd, J= 4.45, $13.51 \mathrm{~Hz}, 1 \mathrm{H}, \mathrm{H}-6$ ), 3.46 (d, J= $13.00 \mathrm{~Hz}, 1 \mathrm{H}, \mathrm{NCH}_{2} \mathrm{Ph}$ ), 3.78 (s, 3H, $\mathrm{OCH}_{3}$ ), 4.02 (d, J= $13.00 \mathrm{~Hz}, 1 \mathrm{H}, \mathrm{NCH}_{2} \mathrm{Ph}$ ), 4.08 (m, 1H, H-3), 6.80-7.40 (m, 9H, Ph-H) ppm; ${ }^{13} \mathrm{C}-\mathrm{NMR}$ (125 Mz, $\mathrm{CDCl}_{3}$ ) $\delta: 32.34$ (1C), 37.75 (1C), 51.49 (1C), 55.24 (1C), 58.86

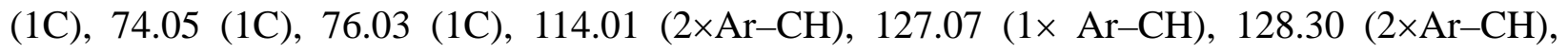

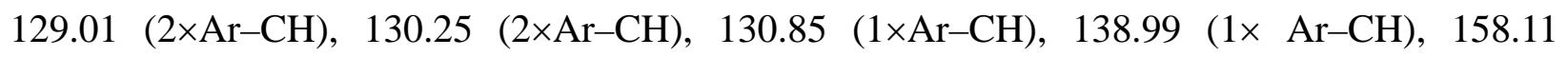
(1×Ar-CH) ppm. MS (EI): $297\left(\mathrm{M}^{+}, 9\right), 279\left(\mathrm{M}^{+}-\mathrm{H}_{2} \mathrm{O}, 69\right), 277$ (3.19), 176(100), 91(92.24); HRMS calcd for $\left[\mathrm{C}_{11} \mathrm{H}_{14} \mathrm{NO}\left(\mathrm{M}^{+}-\mathrm{p}-\mathrm{CH}_{3} \mathrm{OC}_{6} \mathrm{H}_{4} \mathrm{CH}_{2}\right)\right]$ 176.1075, found 176.1072.

(S)-1-Benzyloxycarbonyl-3-hydroxy-2-(4-methoxybenzyl)-pyrrolidine (12). To a mixture of 10 (460 mg, $1.55 \mathrm{mmol}$ ) and 10\% Pd-C (187 mg) was added ethanol (8 mL). The mixture was stirred at room temperature and under an atmosphere of $\mathrm{H}_{2}$ for 15 hours. The mixture was filtered over celite, washed with ethyl acetate (1\% $\mathrm{NH}_{3}$ aq.). After concentrated under reduced pressure, the crude pyrrolidine $\mathbf{1 1}$ was obtained as a yellow oil (300 mg, yield, 93.7\%), which was used in the next step as it was.

To an anhydrous $\mathrm{CH}_{2} \mathrm{Cl}_{2}(7 \mathrm{~mL})$ solution of the crude pyrrolidine $\mathbf{1 1}$ and a catalytic amount of DMAP, was successively added $\mathrm{Et}_{3} \mathrm{~N}(0.4 \mathrm{~mL}, 2.9 \mathrm{mmol})$ and $\mathrm{CbzCl}(0.6 \mathrm{~mL}, 1.25 \mathrm{mmol})$ dropwise at $0{ }^{\circ} \mathrm{C}$ and under an atmosphere of $\mathrm{N}_{2}$. The mixture was stirred at room temperature for 4 hours. After concentrated at reduced pressure, the residue was purified by flash chromatography on silica gel (eluent: ethyl acetate: petroleum ether =1:1) affording 12 (409 mg , yield, 82.8\%) as a colorless oil. $[\alpha]_{\mathrm{D}}{ }^{20}+17.7$ (c 1.1, $\mathrm{CHCl}_{3}$ ). IR (film) $v_{\max }$ : 3320, 2950, 1675, 1510, 1410, 1360, 1248, 1105, 1030, 980, $690 \mathrm{~cm}^{-1} .{ }^{1} \mathrm{H}-\mathrm{NMR}$ (500 Mz, $\mathrm{CDCl}_{3}$ ) (show the peaks of two rotamers) $\delta$ : 1.75-1.95 (2m, 2H, H-4), 2.45 (m, 1H, H-2), 2.95, 3.12 (2dd, J= 3.30, 13.66; 2.80, 13.66, 1H, H-6), 3.47, 3.61 (2m, 2H, H-5), 3.76, 3.78 (2s, 3H, OMe), 3.92, 3.93 (2dd, J= 2.61, 13.66; 2.12, 13.66 Hz, 1H, H-6), 4.16 (s br, 1H, H-3), 5.10-5.22 (m, 2H, NCH $\mathrm{Nh}_{\text {), }}$ 6.70-7.40 (m, 9H, Ph-H) ppm; ${ }^{13} \mathrm{C}-\mathrm{NMR}\left(125 \mathrm{Mz}, \mathrm{CDCl}_{3}\right.$ ) (show the peaks of two rotamers) $\delta$ : 
30.94, 31.49 (1C), 37.38, 38.43 (1C), 44.41, 44.65 (1C), 55.24 (C-3), 66.70, 67.10 (1C), 67.81, 68.36 (1C), 73.46, 74.36 (1C), 113.99(2×Ar-CH), 127.83, 127.95 (1×Ar-CH), 128.06 $(2 \times \mathrm{Ar}-\mathrm{CH}), 128.50(2 \times \mathrm{Ar}-\mathrm{CH}), 129.97(2 \times \mathrm{Ar}-\mathrm{CH}), 130.14(1 \times \mathrm{Ar}-\mathrm{CH}), 130.34(2 \times \mathrm{Ar}-\mathrm{CH})$, 136.68, $136.93(1 \times \mathrm{Ar}-\mathrm{CH}), 155.09(1 \times \mathrm{Ar}-\mathrm{CH}), 158.28(\mathrm{C}=\mathrm{O}) \mathrm{ppm} . \mathrm{MS}(\mathrm{EI}): 341\left(\mathrm{M}^{+}, 6.09\right)$, 220 (18.88), 176 (25.25), 121 (19.29), 91 (100); HRMS calcd for $\left[\mathrm{C}_{20} \mathrm{H}_{23} \mathrm{NO}_{4}\right]$ 341.1627, found 341.1628 .

( 2R, 3S ) - 1 - ( Benzyloxycarbonyl ) - 2 - ( 4-methoxybenzyl ) pyrrolidin - 3 - yl S-Methyl Xanthate (13). To a suspension of $\mathrm{NaH}(170 \mathrm{mg}, 50 \%$ in petrolatum, $3.53 \mathrm{mmol}$ ) and imidazole $\left(75 \mathrm{mg}\right.$ ) in anhydrous THF $(2.0 \mathrm{~mL})$, was added, under an atmosphere of $\mathrm{N}_{2}$, a solution of 12 (370 mg, $1.08 \mathrm{mmol}$ ) in THF ( $5 \mathrm{~mL}$ ). After stirred at $60{ }^{\circ} \mathrm{C}$ for 2 hours, carbon disulfide $(0.3 \mathrm{~mL}, 6 \mathrm{mmol})$ was added dropwise. The mixture was stirred at $60{ }^{\circ} \mathrm{C}$ for 4 hours. Then, methyl iodide $(0.3 \mathrm{~mL}, 4.8 \mathrm{mmol})$ was added, and the mixture was refluxed for additional 4 hours. The mixture was cooled with an ice-bath, then quenched by water $(4 \mathrm{~mL})$. The reaction mixture was extracted with ethyl acetate $(3 \times 10 \mathrm{~mL})$. The combined organic phase was washed with brine, dried with anhydrous $\mathrm{Na}_{2} \mathrm{SO}_{4}$. After concentrated at reduced pressure, the residue was purified by flash chromatography on silica gel (eluent: ethyl acetate: petroleum ether $=1: 5$ ) to yield 13 (286 $\mathrm{mg}$, yield, $72 \%$ ) as a yellow oil. $[\alpha]_{\mathrm{D}}{ }^{20}-3.3$ (c 1.5, $\mathrm{CHCl}_{3}$ ). IR (film) $v_{\max }$ : 2950, 1700, 1510, 1410, 1345, 1250, 1210, 1100, 1040, $690 \mathrm{~cm}^{-1} .{ }^{1} \mathrm{H}-\mathrm{NMR}\left(500 \mathrm{Mz}, \mathrm{CDCl}_{3}\right.$ ) (show the peaks of two rotamers) $\delta: 1.70(\mathrm{~m}, 1 \mathrm{H}, \mathrm{H}-4), 2.04(\mathrm{~m}, 1 \mathrm{H}, \mathrm{H}-4), 2.50\left(\mathrm{~s}, 3 \mathrm{H}, \mathrm{S}-\mathrm{CH}_{3}\right), 2.78$, 2.90 (2dd, J= 7.52, 13.82; 7.67, 13.82, 1H, H-6), 2.95, 3.07 (2dd, J= 3.68, 13.82; 3.15, $13.82 \mathrm{~Hz}$, 1H, H-6), 3.40- 3.62 (m, 2H, H-5 ), 3.77, 3.79 (2s, 3H, OMe), 4.22, 4.31 (2dd, J= 3.15, 3.68, 7.52, 7.67 Hz, 1H, H-2), 5.16, 5.24 (2dd, J=12.10, 12.59; 12.10, $12.59 \mathrm{~Hz}, 2 \mathrm{H}, \mathrm{NCH}_{2} \mathrm{Ph}$ ), 5.75 (m, 1H, H-3), 6.70-7.40 (m, 9H, Ph-H) ppm; ${ }^{13} \mathrm{C}-\mathrm{NMR}\left(125 \mathrm{Mz}, \mathrm{CDCl}_{3}\right.$ ) (show the peaks of two rotamers) $\delta: 19.06$ (S-C), 28.41, 29.24 (1C), 36.35, 37.59 (1C), 44.85, 45.19 (1C), 55.23 (C-3), 64.54, 64.96 (1C), 66.83, 67.23 (1C), 85.67, 86.42 (1C), 113.96, 113.99(2×Ar-CH), 127.85 (1×Ar-CH), 128.06, $128.13(2 \times \mathrm{Ar}-\mathrm{CH}), 128.54(2 \times \mathrm{Ar}-\mathrm{CH}), 128.97,129.16(1 \times \mathrm{Ar}-\mathrm{CH}), 130.42$, $130.58(2 \times \mathrm{Ar}-\mathrm{CH}), 136.55,136.86(1 \times \mathrm{Ar}-\mathrm{CH}), 154.71,154.82(1 \times \mathrm{Ar}-\mathrm{CH}), 158.28(\mathrm{C}=\mathrm{O})$, 214.75 (C=S) ppm. MS (EI): 431 (M+1, 8.9), 323 (19.5), 310 (33.9), 159 (19.29), 91 (100).

(R)-1-Benzyloxycarbonyl-2-(4-methoxybenzyl)-3-pyrroline (14). Neat 13 was heated to 190 $200{ }^{\circ} \mathrm{C}$ under reduced pressure $(<10 \mathrm{mmHg}$ ) for 2 hours. The resulting residue was purified by flash chromatography on silica gel (eluent: ethyl acetate: petroleum ether $=1: 8$ ) affording pyrroline $14\left(131 \mathrm{mg}\right.$, yield, 87.3\%) as a yellow solid. $[\alpha]_{D}{ }^{20}-214\left(c 1.18, \mathrm{CHCl}_{3}\right)$. lit. $[\alpha]_{D}{ }^{20}$ +1.4 (e.e. $=90 \%)$ for $(S)-14 ;^{8 \mathrm{~h}}[\alpha]_{\mathrm{D}}-190.4\left(c 1.0, \mathrm{CHCl}_{3}\right) ;^{81}[\alpha]_{\mathrm{D}}{ }^{25}-199\left(c 1.17, \mathrm{CHCl}_{3}\right){ }^{8 \mathrm{~m}}[\alpha]_{\mathrm{D}}{ }^{20}$ -135.7 (c 0.76, $\left.\mathrm{CHCl}_{3}\right),{ }^{10}$ for (R)-14]. IR (film) $v_{\max }$ : 2900, 1700, 1610, 1510, 1410, 1360, 1320, 1245, 1100, 1025, $690 \mathrm{~cm}^{-1} .{ }^{1} \mathrm{H}-\mathrm{NMR}\left(500 \mathrm{Mz}, \mathrm{CDCl}_{3}\right.$ ) (show the peaks of two rotamers) $\delta: 2.78$, 2.90 (2dd, J= 7.98, 13.21; 8.06, 13.20, 1H, H-6), 3.02, 3.13 (2dd, J= 3.10, 13.20; 3.07, $13.21 \mathrm{~Hz}$, 1H, H-6), 3.76, 3.78 (2s, 3H, OMe), 3.80, 3.83 (2m, 1H, H-5), 4.14, 4.20 (2dm, 1H, H-5), 4.75, 4.80 (2m, 1H, H-2), 5.20, 5.25 (2dd, J=12.08, 12.51; 12.08, $12.51 \mathrm{~Hz}, 2 \mathrm{H}, \mathrm{NCH}_{2} \mathrm{Ph}$ ), 5.60-5.72 (m, 1H, H-3), 6.70-7.50 (m, 9H, Ph-H ) ppm; ${ }^{13} \mathrm{C}-\mathrm{NMR}\left(125 \mathrm{Mz}, \mathrm{CDCl}_{3}\right.$ ) (show the peaks of two rotamers) $\delta$ : 38.31, 39.73 (1C), 53.56, 53.99 (1C), 55.18 (C-3), 65.36, 65.87 (1C), 66.55, 
67.06 (1C), 113.44, $113.54(2 \times \mathrm{Ar}-\mathrm{CH}), 125.29,125.53(=\mathrm{CH}), 127.86,127.94(1 \times \mathrm{Ar}-\mathrm{CH})$, 128.10, 128.16 (2×Ar-CH), 128.48, 128.57 (2×Ar-CH), 129.40, $129.49(=\mathrm{CH}) 129.57,129.63$ (1×Ar-CH), 130.59, 130.79 (2×Ar-CH), 136.78, 137.08 (1×Ar-CH), 154.41, $154.65(1 \times \mathrm{Ar}-\mathrm{CH})$, 158.04, 158.11 (C=O) ppm. MS (EI): $323\left(\mathrm{M}^{+}, 16.1\right), 219$ (7.3), 202 (29.0), 158 (35.5), 91(92.24); HRMS calcd for $\left[\mathrm{C}_{12} \mathrm{H}_{12} \mathrm{NO}_{2}\left(\mathrm{M}-\mathrm{CH}_{3} \mathrm{OC}_{6} \mathrm{H}_{4} \mathrm{CH}_{2}\right)\right]$ 202.0907, found 202.0868.

\section{Acknowledgements}

The authors are grateful to the National Science Fund for Distinguished Young Investigators (29625204), the NSF of China (29832020, 20072031), Qiu Shi Science \& Technologies Foundation, Foundation for University Key Teacher by the Ministry of Education and Xiamen University for financial support.

\section{References}

1. Sobin, B. A.; Tanner, F. W., Jr. J. Am Chem. Soc. 1954, 76, 4053.

2. Jimenez, A.; Vazquez, D. in Antibiotics, ed, F. E. Hahn, Springer Verlag, Berlin, 1979, pp.1-19; For a recent example, see: (a) Dudai, Y. Nature 2000, 406, 686. (b) Nader, K.; Schafe, G. E.; Le Doux, J. E. Nature 2000, 406, 722.

3. Frye, W. W.; Mule, J. G.; Swartzwelder, C. Antibiot. Annu. 1954-1955, 820.

4. Korzybsky, T.; Kowszyk-Gindifer, Z.; Kurgtowicz, W. in Antibiotics, American Society of Microbiology, Washington DC, 1978, pp. 343-346.

5. The Merck Index, 12th ed., Windholz, M., Ed., Merck: Whitehouse Station, NJ, 1996, p 710.

6. Hosoya, Y.; Kameyama, T.; Naganawa, H.; Okami, Y.; Takeuchi, T. J. Antibiot. 1993, 46, 1300.

7. (a)Van der Bosch, J.; Rueller, S.; Schlaak, M. German Patent DE 19,744,676, Chem. Abstr. 1999, 130, 291581. (b) Rueller, S.; Stahl, C.; Kohler, G.; Eickhoff, B.; Breder, J.; Schlaak, M.; van der Bosch, J. Clin. Cancer Res. 1999, 5, 2714.

8. (a) Wong, C. M.; Bucchini, J.; Chang, I.; Te Raa, J.; Schenk, R. Can. J. Chem. 1969, 47, 2421. (b) Oida, S.; Ohki, E. Chem. Pharm. Bull. 1969, 17, 1405. (c) Felner, I.; Schenker, E. Helv. Chim. Acta 1970, 53, 754. (d) Verheyden, J. P. H.; Richardson, A. C.; Bhatt, R. S.; Grant, B. D.; Flitch, W. L.; Moffat, J. G. Pure Appl. Chem. 1978, 50, 1363. (e) Schumacher, D. P.; Hall, S. S. J. Am. Chem. Soc. 1982, 104, 6076. (f) Buchanan, J. G.; MacLean, K. A.; Wightman, R. H.; Paulsen, H. J. Chem. Soc., Perkin Trans. 1 1985, 486. (g) Iida, H.; Yamazaki, N.; Kibayashi, C. J. Org. Chem. 1986, 51, 1069. (h) Meyers, A. 
I.; Dupre, B. Heterocycles 1987, 25, 113. (i) Shono, T.; Kise, N. Chem. Lett. 1987, 697. (j) Baer, H. H.; Zamkanei, M. J. Org. Chem. 1988, 53, 4786. (k) Jegham, S.; Das, B. C. Tetrahedron Lett. 1988, 29, 4419. (l) Takano, S.; Iwabuchi, Y.; Ogasawara, K. Heterocycles 1989, 29, 1861. (m) Takahata, H.; Banba, Y.; Tajima, M.; Momose, T. J. Org. Chem. 1991, 56, 240. (n) Ballini, R.; Marcantoni, E.; Petrini, M. J. Org. Chem. 1992, 57, 1316. (o) Ikota, N. Heterocycles 1995, 41, 983. (p) Yoda, H.; Nakajima, T.; Yamazaki, H.; Takabe, K. Heterocycles 1995, 41, 2423. (q) Shi, Z.-C.; Lin, G.-Q. Tetrahedron: Asymmetry 1995, 6, 2907. (r) Kang, S. H.; Choi, H.-W. Chem. Commun. 1996, 1521. (s) Han, G.; LaPorte, M. G.; McIntosh, M. C.; Weinreb, S. M.; Parvez, M. J. Org. Chem. 1996, 61, 9483. (t) Veith, U.; Schwardt, O.; Jaget, V. Synlett 1996, 1181. (u) Delair, P.; Brot, E.; Kanazawa, A.; Greene, A. E. J. Org. Chem. 1999, 64, 1383. (v) Schwartdt, O.; Veith, U.; Gaspard, C.; Jager, V. Synthesis 1999, 1473. (w) Hutin, P.; Haddad, M.; Larcheveque, M. Tetrahedron: Asymmetry 2000, 11, 2547. (x) Hulme, A.N.; Rosser, E.M. Org. Lett. 2002, 4, 265.

9. (a) Huang, P.-Q.; Chen, Q.-F.; Chen, C. -L.; Zhang, H. -K. Tetrahedron: Asymmetry 1999, 10, 3827. (b) Huang, P.-Q.; Wang, S.-L.; Ye, J.-L.; Ruan, Y.-P.; Huang, Y.-Q.; Zheng, H. Tetrahedron 1998, 54, 12547. (c) Huang, P.-Q.; Ye, J.-L.; Chen, Z.; Ruan, Y.-P. Synthetic Commun. 1998, 28, 417. (d) Huang, P.-Q.; Wang, S.-L.; Zheng, H.; Fei, X.-S. Tetrahedron Lett. 1997, 38, 271.

10. Huang, P. Q.; Wang, S. L.; Ruan, Y. P.; Gao, J. X. Nat. Prod. Lett. 1998, 11, 101. In this communication the regioselectivity in the Grignard reagent addition to malimide 4 should be corrected as 80:20, see, Huang, P. -Q.; Wang, S. -L.; Ruan, Y. -P.; Gao, J. -X. corrigendum submitted to Nat. Prod. Lett.

11. (a) Kursanov, D. N.; Parnes, Z. N.; Loim, N. M. Synthesis 1974, 633. (b) Hart, D. J.; Yang, T.-K. J. Org. Chem. 1985, 50, 235. (c) Rolf, D.; Gray, G. R. J. Am. Chem. Soc. 1982, 104, 3539. (d) Burgess, L. E.; Meyers, A. I. J. Org. Chem. 1992, 57, 1656. (e) Yoda, H.; Kitayama, H.; Yamade, W.; Katagiri, T.; Takabe, K.; Tetrahedron: Asymmetry 1993, 4, 1451.

12. For reviews on the $N$-acyliminium chemistry, see, (a) Speckamp, W. N.; Hiemstra, H. Tetrahedron 1985, 41, 4367. (b) Speckamp, W. N.; Moolenaar, M. J. Tetrahedron 2000, 56, 3817. 